\title{
An Ontology-Based Modeling Approach for Developing a Competencies-Oriented Collective Intelligence
}

\author{
Mihaela Brut ${ }^{1}$, Florence Sedes ${ }^{2}$, Toader Jucan ${ }^{1}$, Romulus Grigoras ${ }^{3}$, \\ Vincent Charvillat ${ }^{3}$ \\ ${ }^{1}$ Alexandru Ioan Cuza University of Iasi, Romania, \\ \{mihaela, jucan\}@infoiasi.ro; \\ ${ }^{2}$ Institut de Recherche en Informatique de Toulouse, France, sedes@irit.fr; \\ ${ }^{3}$ ENSEEIHT, France \{romulus.grigoras, charvi\}@enseeiht.fr
}

\begin{abstract}
Expressing the user competences through domain ontology concepts is a prerogative for developing well managed and semantically enriched user profiles, transferable from a system to another. If, supplementary, the available documents are also ontology-based annotated, the add-on value will concern not only the document semantics, but also the relations and dependencies between users and documents. In such approach, the ontology constitutes the binder between peoples, as well as between peoples and documents, and also between documents. Its reasoning support sustains the development of a collective intelligence, as well as the most suitable knowledge sharing, according to the users common competencies. In this article we present such modeling approach, and also some methods for user and document model development, as well as the advantages for personalized and collaborative facilities.
\end{abstract}

\section{Introduction}

An E-learning platform is a real source of knowledge due to the available materials, but also to its users' competences. Our paper goal is to provide a solution for interconnecting these two resource types in order to provide platform users with access to the most suitable documents and collaborators, according to their profile.

The solution considers the same domain ontology set for modeling both the documents annotations and the user profile. After presenting the two models and their development techniques, our paper will expose the modeling approach advantages and applications, followed by conclusions and further work. 


\section{Ontology-Based User Competences Model}

The idea of managing user competencies through one or more ontologies was explored in multiple systems. The CommOn framework uses self-defined competence schemes, organized as ontology, in order to develop Competency-Based Web Services dedicated to Human Resource Management [10]. In [4] is presented an system for storing and reasoning about competencies of students at universities, based on a fine-grained representation of skills and competence ontology.

General user models consider five most popular features for user as an individual: the user's knowledge, interests, goals, background, and individual traits [2]. The overlay model enables to represent these features as a subset of the domain model, which could consist in one or more domain ontologies.

Our goal is to integrate such overlay model focused on competences inside the user model provided by the e-learning standards and to find a solution for automatically developing it. For an e-learning platform aiming the user competences management and development, two features are essential for the user model:

1. Knowledge - the user actual, acquired, competences, expressed through ontology concepts selected according his acquired certificates and qualifications.

2. Interests - the user desired, foresighted, competencies, expressed through ontology concepts selected according the courses in which the students is currently enrolled (each course aims at developing a set of competences);

Among the two main user profile models standardized in the e-learning domain IMS Learner Information Specification and IEEE PAPI Public And Private, we choose PAPI for our user profile model, because we intend to use IEEE LOM standard for structuring the educational content. PAPI model structure includes 6 categories [11], the Learner Performance category being in charge with competence management, including support for tracking the competences level and validity.

Because the two layers user profile development is related to certain events such as exams upholding or course enrolment, a rule-based solution for it's automatically updating is suitable [7]:

- When a user pass an exam and gains a certificate, its associated competences (ontology concepts) are transferred into the user knowledge level, being deleted from the interest layer profile.

- When a user is enrolled to a certain course, the course assigned topics (ontology concepts) are included automatically in his long term interests profile.

\section{The Domain Model}

The IEEE/LOM (Learning Objects Metadata) standard for the educational materials structuring is considered as the most enabling for semantic extensions. Its Classification category enables to specify that the LO belongs to a certain classification system such a domain ontology, by identifying the ontology and the particular concept which want to refer.

For enhancing the expressive power of annotations that create links between documents and ontology concepts, there were conceived some techniques of 
associating roles and/or weights with these relations. The most common role is "prerequisite" [1]. In the KBS-HyperBook system, the documents could be marked as "problem statement", "example", "theory" for a certain concept [6].

Because our goal is to identify the most relevant documents for a certain user competence profile, we propose the usage of 3 roles (as relation types) for expressing the relevance of a document for a certain concept. These could be integrated into Classification IEEE/LOM category as a new attribute, relationType, with 3 possible values expressing this relevance into a decreasing order:

- isOnTopic - for a document which is especially destined to a certain topic;

- usesTheConcept - expressing the concepts encountered into document;

- makesReferenceTo - for marking the other referenced concepts from ontology.

For automatically generating this relations, our solution first distributes the document in 3 classes, which are relevant for our considered relations: the document title and subtitles will be considered for the isOnTopic relation, the hyperlinks and the bibliographical references - for the makesReferenceTo relation, and the document body (the rest of the document) will be processed in order to obtain the usesTheConcept relation. The existing Web Information Retrieval techniques consider a larger number of classes, corresponding for example to 12 categories [7].

For each class concept-based annotation, the existing approaches consider the Latent Semantic Indexing techniques [5], which represent a document through concepts rather than through index terms, as in term-based classical information retrieval. First the matrix of terms frequency is built, and then the matrix of concepts frequency is obtained, by applying the Singular Value Decomposition technique [8]. Thus, for each of the above mentioned 3 document classes, there is obtained the corresponding set of concepts, together with their occurrence number.

\section{The Modeling Approach Advantages}

The documents and users modeling in the exposed manner provides a permanent connection between users and materials which could be exploited for many purposes:

- recommending a user the potential collaborators (in general or in specific projects)

- filtering and ranking the existing materials according to a user profile,

- adapting the navigation system to a user profile (by re-ordering the links, emphasizing the most important ones, or eliminating those which are not useful).

Because each such facility is focused on a sub-set of the ontology competencies set, the selection of the suitable materials, as well as the user selection could be reduced in fact to a query process. Thus, all the mentioned situations could be reduced to the case of querying the users and materials XML databases by a ontology concepts based query. A ranking algorithm processes such queries, considering as most relevant the documents which contain:

- the query concepts linked through strong semantic relations (e.g. isOnTopic);

- the concepts related to the query concepts;

These criteria are adapted from ILEX (The Intelligent Labeling Explorer) system [9]: (i) information becomes less relevant the more distant it is from the focal 
object, in terms of semantic links; (ii) different semantic link types maintain relevance to different degrees.

- which has a good temporal relevance (recently created or updated);

- which author is competent concerning the query concepts.

These facilities could be exported from an e-learning system to another if they are implemented in the form of Web services [3]. Thus, the user mobility is facilitated by his user competencies profile recognition by other systems.

\section{Conclusions and Further Work}

In this article, we presented a modeling approach for the content annotations and user competences profile within e-learning platforms, based on the same domain ontology set. This approach considers all the system resources from the perspective of enhancing user competencies and collaboration, contributing to the collective intelligence development.

A further research direction consists in exploring the implementation and optimization techniques details for the particular case of personalized recommendations, considering also the collaborative filtering criterion.

\section{References}

1. Brusilovsky, P., Eklund, J., Schwarz, E.: Web-based education for all: A tool for developing adaptive courseware. In: Ashman, H., Thistewaite, P. (eds.) Proc. of Seventh International World Wide Web Conference. Vol. 30. Elsevier Science B. V. (1998) 291-300

2. Brusilovsky, P., Millán, E., User Models for Adaptive Hypermedia and Adaptive Educational Systems, in P. Brusilovsky, A. Kobsa, W. Nejdl (Eds.): The Adaptive Web, LNCS 4321, Springer 2007

3. Brut, M., Buraga, S., Ontology-Based Annotation Grid Services for E-Learning, in Xhafa F., Barolli, L., Proceedings of Conference on Complex, Intelligent and Software Intensive Systems, IEEE Computer Society Press, ISBN 0-7695-3109-1, 2008

4. Dorn, J., Pichlmair, M., A Competence Management System for Universities, Proceeding of the European Conference on Artificial Intelligence, St. Gallen, 2007

5. Dumais, S.T.: Latent semantic indexing (LSI) and TREC-2. In: Text REtrieval Conference (TREC) TREC-2 Proceedings (1993) 105-116 NIST Special Publication 500-215.

6. Henze, N., Nejdl, W.: Adaptation in open corpus hypermedia. International Journal of Artificial Intelligence in Education 12, 4 (2001) 325-350

7. Lin, W., Alvarez, S.A., Ruiz, C.: Efficient adaptive-support association rule mining for recommender systems. Data Mining and Knowledge Discovery 6 (2002) 83-105

8. Micarelli, A., Sciarrone, F., Marinilli, M.: Web document modeling. In Brusilovsky, P., Kobsa, A., Nejdl, W., eds.: The Adaptive Web, LNCS 4321, Springer 2007

9. O'Donnell, M., Mellish, C., Oberlander, J., Knott, A.: ILEX: An Architecture for a Dynamic Hypertext Generation System. Journal of Natural Language Engineering 7(3) (2003)

10. Trichet, Leclere, A Framework for building competency-based systems, in N. Zhong et al. (Eds.): ISMIS 2003, LNAI 2871, pp. 633-639, Springer, 2003.

11. ***, IEEE P1484.2.25 - Draft Standard for Learning Technology. Public and Private Information (PAPI) for Learners (PAPI Learner) Learner Performance Information, 2001 